Differential associations of cardiac troponin $\mathrm{T}$ and cardiac troponin I with coronary artery pathology and dynamics in response to short-duration exercise

\title{
Authors
}

Sjur H. Tveit, MD ${ }^{\mathrm{a}, \mathrm{b}, \mathrm{c}}$; Joanna Cwikiel MD ${ }^{\mathrm{b}, \mathrm{c}, \mathrm{d}}$; Peder L. Myhre MD, PhD ${ }^{\mathrm{a}, \mathrm{b}, \mathrm{c}}$; Torbjørn Omland MD, PhD, MPH, FACC ${ }^{\text {a,b }}$; Eivind Berge MD, PhD ${ }^{\dagger, d}$; Ingebjørg Seljeflot PhD, FESC $^{\mathrm{b}, \mathrm{c}, \mathrm{d}}$; Arnljot Flaa MD, $\mathrm{PhD}^{\mathrm{d}, \mathrm{e}}$

\section{Affiliations and institutions}

aDivision of Medicine, Department of Cardiology, Akershus University Hospital, Lørenskog, Norway

${ }^{\mathrm{b}}$ Institute of Clinical Medicine, Faculty of Medicine, University of Oslo, Oslo, Norway

${ }^{c}$ Center for Clinical Heart Research, Department of Cardiology, Oslo University Hospital Ullevål, Oslo, Norway

${ }^{\mathrm{d}}$ Division of Medicine, Department of Cardiology, Oslo University Hospital Ullevål, Oslo, Norway

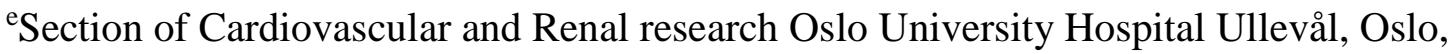
Norway

†'Deceased

\section{Corresponding author}

Sjur Hansen Tveit, MD

Department of Cardiology, Akershus University Hospital, Lørenskog, Norway

NO-1478 Lørenskog, Norway

Tel: +4793011508 e-mail: sjur.hansen.tveit@ahus.no 


\section{Declaration of interest:}

T. Omland has received speaker's honoraria from Roche Diagnostics, as well as research support from Roche Diagnostics and Singulex Inc. None of the other authors have reported disclosures pertaining to this work.

\section{Funding:}

The CADENCE study received financial support from the University of Oslo and the Stein Erik Hagen Foundation for Clinical Heart Research. Singulex Inc. contributed reagents for the hs-cTnI assay employed in the study. 


\section{ABSTRACT}

\section{Background}

We aimed to assess the associations between cardiac troponin (cTn) T and I concentrations, physical exercise and the presence and severity of angiographic coronary artery disease (CAD) in patients evaluated for suspected chronic coronary syndrome (CCS).

\section{Methods and results}

All patients performed an exercise stress test on a bicycle ergometer and underwent invasive coronary angiography with weighted anatomical evaluation using the Gensini score. Blood samples were collected before and after exercise and analysed with high-sensitivity (hs) cTnT and cTnI assays.

Of 297 patients (median age 62 (Quartile [Q]1-3 56-69) years, 35\% female), 46\% were categorized as "severe CAD” (Gensini score $\geq 20)$.

Resting hs-cTnT and hs-cTnI concentrations were detectable in $88 \%$ and $100 \%$ of patients, with medians of 6 (Q1-3 4-9) ng/L and 1.5 (0.9-2.4) ng/L, respectively.

In adjusted normalized linear regression analyses, higher resting concentrations were associated with increasing Gensini score (hs-cTnT: B 0.19, 95\% Confidence Interval [CI] [0.09-0.41], $p<0.001$; hs-cTnI: B 0.18, [0.06-0.30], $p=0.002)$.

The area under the receiver operating characteristics curve for predicting severe CAD was $0.72(95 \%$ CI [0.66-0.78]) and $0.68(0.62-0.74)$ for resting hs-cTnT and hs-cTnI, $p=0.11$ for difference.

The median (Q1-3) relative increase in hs-cTnT and hs-cTnI concentrations were $5(0-12) \%$ and 13 (3-27) \%, respectively, with no significant associations with CAD severity. 


\section{Conclusions}

In patients with suspected CCS, higher hs-cTn concentrations at rest were associated with increasing angiographic severity of $\mathrm{CAD}$, without any significant differences between the troponin isotypes. Post-exercise hs-cTn concentrations did not have discriminatory power for CAD. 


\title{
Keywords
}

Stable coronary artery disease; Chronic coronary syndrome; High-sensitivity; Troponin;

Exercise testing

\begin{abstract}
Abbreviations
cTnI - Cardiac troponin isotype I; cTnT - Cardiac troponin isotype T; hs-cTn - Highsensitivity cardiac troponin; CAD - Coronary artery disease; CCS - Chronic coronary syndrome; CV - Coefficient of variation; URL - Upper Reference Limit; LoD - Limit of Detection; LoB - Limit of Blank
\end{abstract}




\section{INTRODUCTION}

The two cardiac specific troponin isotypes I and T (cTnI, cTnT) are used interchangeably in the diagnosis of myocardial injury. In the setting of acute coronary syndromes (ACS), elevated and dynamic concentrations above the assay-specific $99^{\text {th }}$ percentile upper reference limit (URL) signify acute myocardial infarction (AMI). In chronic coronary syndromes (CCS), elevated but non-dynamic concentrations signify chronic myocardial injury. [1] In patients with CCS, there is a graded relationship between the concentrations of cTn and the extent and severity of coronary lesions. [2,3] This is not limited to concentrations above the $99^{\text {th }}$ percentile URL but is also observed within the reference range of normality. [2] In these patients, cTn has been demonstrated to be a robust marker of poor prognosis, both for cardiovascular events and all-cause mortality. $[4,5]$

With the advent of high-sensitivity cTn (hs-cTn) assays, it has become possible to detect and quantify cTn at lower concentrations and with greater analytical accuracy than with previous generations of cTn assays. However, due to different epitope specificity of antibodies used in different assays [6] and to different reference populations, there is considerable inter-assay variability in URLs. As such, direct comparison of cTn concentrations between different assays in a clinical setting remains problematic. However, due to the increased analytical accuracy at lower concentrations, previously undetectable increases in cTn are now observed in response to physical exercise [7]. Recent studies indicate that this phenomenon, rather than being benign, is of possible diagnostic and prognostic importance. $[8,9]$

Although cTnI and cTnT are used interchangeably in the diagnosis of AMI, there is evidence that the two isotypes are differentially associated with several physiological and pathophysiological processes. [10, 11] For instance, in contrast to cTnI, cTnT shows diurnal variation and seems to be a more robust marker of poor long-term outcome [12-14], whereas cTnI increases earlier and with greater magnitude in the setting of myocardial ischemia [12] 
and is less associated with renal function than cTnT. [15] Any clinical implications of these phenomena have yet to be established.

In this study, we aimed to assess the relative strength of associations between resting concentrations of $\mathrm{cTnI}$ and $\mathrm{cTnT}$ and their dynamic response to short duration exercise and the angiographic extent and severity of coronary artery disease (CAD) in patients with suspected CSS. 


\section{MATERIALS AND METHODS}

\subsection{Study design}

The current investigation utilizes data from the Markers of Coronary Artery Disease During Exercise Testing (CADENCE) study (NCT01495091). Results from this study have previously been published. [16] Briefly, patients referred for evaluation of suspected CCS, 18 years or older and a Morise score $\geq 9$ (i.e. intermediate or high pre-test CAD risk [17]) were eligible for inclusion. The study was conducted at Oslo University Hospital, Ullevål (OUH) and written informed consent was obtained prior to inclusion. Major exclusion criteria included ACS, symptomatic heart failure (HF), moderate to severe valvular heart disease, previous coronary artery bypass graft $(\mathrm{CABG})$, ongoing cardiac dysrhythmias such as atrial fibrillations, renal insufficiency (estimated glomerular filtration rate $<45 \mathrm{ml} / \mathrm{min} / 1.73 \mathrm{~m}^{2}$ ) or inability to follow study protocol. All patients underwent a baseline physical examination, cardiac exercise stress test on an upright stationary ergometer bicycle and invasive coronary angiography. History of hypertension was defined as known diagnosis or use of antihypertensive medication. History of CAD was defined as previous AMI or coronary revascularization. The study was approved by the Regional Ethics Committee and performed in accordance with the Declaration of Helsinki.

\subsection{Cardiac exercise stress test}

Cardiac exercise stress test was performed on an upright stationary ergometer bicycle (CS-200 Excellence, Switzerland or Ergoline, Germany) supervised by a physician and nurse team. All patients were monitored with 12-lead ECG for the duration of the test. Any dynamic STsegment elevation, horizontal or downward sloping ST-segment depression $>0.1 \mathrm{mV} 60 \mathrm{~ms}$ after the J-point and/or ischemic symptoms were defined as a positive test. All patients were exercised to exhaustion, starting at $30 \mathrm{~W}$ for women and $50 \mathrm{~W}$ for men with a gradual increase of $10 \mathrm{~W}$ per min. The patient target pedal cadence was set at 65 rotations per min. Blood 
pressure was measured manually every 5 min and patients asked to grade their perceived exertion using the Borg scale [18] in addition to the calculated metabolic equivalent of task (MET). The test was terminated if the patients developed ischemic symptoms or signs on ECG, cardiac arrhythmias, inability to maintain exercise intensity or insufficient or exaggerated chronotropic or blood pressure response. Ten percent of test results were assessed by an independent physician with a $100 \%$ concordance rate with the primary test physician.

\subsection{Invasive coronary angiography}

Invasive coronary angiography was performed by trained interventional cardiologists using standard Seldinger technique with primarily radial artery access. All coronary segments were retrospectively described and graded according to a modified version of the American Heart Association coronary segmentation definition using the Gensini score. [19, 20] In cases of doubt (20\%) a second blinded interventional cardiologist re-evaluated the angiograms with an inter-observer variability of $7 \%$. The Gensini score provides a graded assessment of the total coronary artery plaque burden based on a weighted grading of separate coronary artery segments. The primary end point of severe $C A D$ was defined as a Gensini score $\geq 20$, corresponding in severity to an angiographically verified singular left main coronary artery stenosis $\geq 70 \%$. Non-severe $C A D$ was defined as Gensini score 1-19 and No CAD as Gensini score 0.

\subsection{Blood sampling and biochemical assays}

Blood samples were obtained by venepuncture prior to the exercise stress test, and within five min after completion of the test. Serum was obtained by centrifugation at $2500 \mathrm{x} g$ for $10 \mathrm{~min}$ at room temperature and kept frozen at $-80^{\circ} \mathrm{C}$ until analysed. Routine analyses were performed by conventional laboratory methods. cTnI was measured at Akershus University Hospital with the Singulex Clarity Single Molecule Counting (SMC) ultra-sensitivity cTnI assay. As reported by the manufacturer, this assay has a limit of blank 
$(\mathrm{LoB})$ at $0.02 \mathrm{ng} / \mathrm{L}$, Limit of detection $(\mathrm{LoD})$ at $0.08 \mathrm{ng} / \mathrm{L}$ and a $99^{\text {th }}$ percentile URL of 8.67 $\mathrm{ng} / \mathrm{L}$. The coefficient of variation $(\mathrm{CV})$ was $14.5 \%$ at a sample concentration of $0-2.00 \mathrm{ng} / \mathrm{L}$, $11.5 \%$ at $10.00 \mathrm{ng} / \mathrm{L}$ and $6.8 \%$ at $100.00 \mathrm{ng} / \mathrm{L}$. cTnT was analysed at OUH with the Roche fifth generation hs-cTnT assay (Gen 5 STAT cardiac troponin T) on a Cobas e602 platform. Per the manufacturer, this assay has a LoB at $3 \mathrm{ng} / \mathrm{L}$, LoD at $5 \mathrm{ng} / \mathrm{L}$ and a $99^{\text {th }}$ percentile URL of $14 \mathrm{ng} / \mathrm{L}$. The inter-assay CV is reported to be $7.8 \%$ at a sample concentration of $6.5 \mathrm{ng} / \mathrm{L}$ and $4.8 \%$ at a sample concentration of $11.5 \mathrm{ng} / \mathrm{L}$. In our laboratory the $\mathrm{CV}$ was $6 \%$ at a sample concentration of $13 \mathrm{ng} / \mathrm{L}$. To allow for an assumed but unmeasurable distribution, hscTnT concentrations below the $\mathrm{LoB}$ are imputed as $1.5 \mathrm{ng} / \mathrm{L}$ in all analyses. To utilize the high analytical sensitivity and precision of the SMC hs-cTnI assay, concentrations are reported to one decimal place, while hs-cTnT concentrations are reported as whole numbers as recommended by the International Federation of Clinical Chemistry and Laboratory Medicine (IFCC). The Chronic Kidney Disease Epidemiology Collaboration (CKD-EPI) equation was utilized to calculate the estimated glomerular filtration rate (eGFR).

\subsection{Statistical analysis}

Continuous variables are reported as medians with quartiles 1 and 3 (Q1-3), categorical variables as absolute numbers with percentages. We use non-parametric tests when assessing baseline variables; Cuzick's non-parametric trend test for continuous variables and chi-square trend test for categorical variables. Spearman's rank correlation was utilized to assess correlates of each troponin isotype. Predictors of resting hs-cTn, exercise-induced changes in hs-cTn, and the Gensini score were identified using linear regression analyses. Logistic regression models were used to analyse predictors of severe CAD. In adjusted regression analyses, the following variables were selected a priori; age, sex, body mass index (BMI), current smoking, systolic blood pressure, history of diabetes, history of CAD and eGFR. All variables were assessed for univariable associations followed by multivariable modelling of 
significant predictors utilizing a stepwise backward elimination approach. To account for a right-skewed distribution, we use base 2 logarithmically transformed values of hs-cTnI and hs-cTnT in all regression models. Exercises induced changes in cTn concentrations are expressed as ratios and are also logarithmically transformed when included in regression models. Due to the semi-continuous distribution of the Gensini score, transformation to normality is not possible. Thus, in regression analyses, we modelled the Gensini score either as a dichotomous variable (i.e. zero vs. non-zero), or the non-zero scores as a base 2 logarithmically transformed continuous variable. To compare regression coefficients and odds ratios between hs-cTnI and hs-cTnT we normalized the logarithmically transformed covariates. Finally, to assess the differential performance of hs-cTnI and hs-cTnT in predicting severe $\mathrm{CAD}$ we compared the areas under the receiver operating characteristics curves (ROC-AUC), as well as the Continuous Net Reclassification Improvement (cNRI) and Integrated Discrimination Improvement (IDI) with cTn added to a base risk prediction model consisting of age, sex, history of CAD, diabetes, hypertension, low-density lipoprotein (LDL) cholesterol concentrations and current smoking.

All statistical analyses were performed with STATA 15 (StataCorp. 2017. Stata Statistical Software: Release 15.1. College Station, TX: StataCorp LLC). p-values with a significance level of $5 \%$ or confidence intervals (CI) with a confidence level of $95 \%$ are used to indicate assumed statistical significance. 


\section{RESULTS}

\subsection{Baseline characteristics}

In total, 297 patients were included in the study, with median age 62 (Q1-3 56-69) years, 105 (35\%) women and $119(40 \%)$ with a history of CAD. The median Gensini score was 17.5 (Q1-3 6.0-43.5). When categorizing patients based on Gensini score, 25 (8\%) had no CAD $($ Gensini score $=0), 135$ (46\%) had non-severe CAD (Gensini score 1-19) and 137 (46\%) had severe CAD (Gensini score $\geq 20$ ). Increasing severity of CAD was associated with older age, male sex, history of hypertension or CAD and use of antiplatelet drugs, statins and beta/calcium channel blockers. With increasing severity of CAD, patients also had lower LDLcholesterol, lower eGFR and higher NT-proBNP concentrations. (Table 1) The median exercise duration was 9.5 (Q1-3 7.5-12.1) min and the median MET was 6.7 (Q1-3 5.6-8.0); $p$ for trend $=0.02$ and 0.04 , respectively, with increasing severity of CAD. 
Table 1. Baseline characteristics of patients referred for evaluation of suspected chronic coronary syndrome stratified by categories of Gensini score.

\begin{tabular}{|c|c|c|c|c|}
\hline & $\begin{array}{l}\text { No coronary } \\
\text { artery disease } \\
(\text { Gensini }=0) \\
\quad(n=25)\end{array}$ & $\begin{array}{c}\text { Non-severe } \\
\text { coronary artery } \\
\text { disease } \\
\text { (Gensini }=1-19) \\
\quad(n=135)\end{array}$ & $\begin{array}{l}\text { Severe coronary } \\
\text { artery disease } \\
\text { (Gensini } \geq 20) \\
\quad(n=137)\end{array}$ & $\begin{array}{l}p \text {-value for } \\
\text { trend }\end{array}$ \\
\hline Age (years) & $57(53,64)$ & $62(54,68)$ & $64(58,69)$ & 0.002 \\
\hline Female sex & $15(60 \%)$ & $67(50 \%)$ & $23(17 \%)$ & $<0.001$ \\
\hline Family history of CAD & $12(48 \%)$ & $78(57 \%)$ & $58(42 \%)$ & 0.07 \\
\hline Current smoking & $7(28 \%)$ & $24(18 \%)$ & $22(16 \%)$ & 0.24 \\
\hline Body Mass Index $\left(\mathrm{kg} / \mathrm{m}^{2}\right)$ & $28(26,31)$ & $26(24,29)$ & $27(25,30)$ & 0.79 \\
\hline Systolic blood pressure (mmHg) & $126(115,136)$ & $130(119,148)$ & $139(126,154)$ & $<0.001$ \\
\hline Heart rate & $68(62,75)$ & $70(63,78)$ & $67(59,76)$ & 0.10 \\
\hline \multicolumn{5}{|l|}{ History of } \\
\hline Hypertension & $12(48 \%)$ & $72(53 \%)$ & $92(67 \%)$ & 0.01 \\
\hline Diabetes type I or II & $7(28 \%)$ & $22(16 \%)$ & $27(20 \%)$ & 0.79 \\
\hline Coronary artery disease & $0(0 \%)$ & $28(21 \%)$ & $61(45 \%)$ & $<0.001$ \\
\hline \multicolumn{5}{|l|}{ Medication } \\
\hline Acetylsalicylic acid (ASA) & $9(36 \%)$ & $86(64 \%)$ & $119(87 \%)$ & $<0.001$ \\
\hline Clopidogrel & $0(0 \%)$ & $5(4 \%)$ & $14(10 \%)$ & 0.01 \\
\hline Warfarin & $0(0 \%)$ & $2(2 \%)$ & $3(2 \%)$ & 0.43 \\
\hline Statins & $10(40 \%)$ & $89(66 \%)$ & $113(83 \%)$ & $<0.001$ \\
\hline Beta blocker & $2(8 \%)$ & $51(38 \%)$ & $70(51 \%)$ & $<0.001$ \\
\hline Calcium blocker & $1(4 \%)$ & $15(11 \%)$ & $32(23 \%)$ & 0.001 \\
\hline ACE-i or ARB & $9(36 \%)$ & $40(30 \%)$ & $59(43 \%)$ & 0.08 \\
\hline Exercise duration (min) & $10.3(8.7,12.7)$ & $9.7(7.8,12.2)$ & $9.2(6.6,11.9)$ & 0.02 \\
\hline METs & $7.0(6.3,7.8)$ & $6.9(5.8,8.0)$ & $6.3(5.5,8.0)$ & 0.04 \\
\hline Hemoglobin concentration (g/dl) & $14.5(14.0,15.2)$ & $14.3(13.4,15.1)$ & $15.05(14.2,15.7)$ & $<0.001$ \\
\hline White blood cell count $\left(\times 10^{9} / \mathrm{L}\right)$ & $6.8(5.8,8.5)$ & $6.4(5.6,7.7)$ & $7(6,8.3)$ & 0.10 \\
\hline eGFR ckd-epi (ml/min $\left./ 1.73^{2}\right)$ & $89(81,99)$ & $85(75,96)$ & $83(71,92)$ & 0.02 \\
\hline $\begin{array}{l}\text { Low density lipoprotein } \\
\text { cholesterol (mmol/L) }\end{array}$ & $3.0(2.5,3.8)$ & $2.7(2.2,3.9)$ & $2.4(1.9,3.1)$ & $<0.001$ \\
\hline $\mathrm{HbA1c}(\%)$ & $5.6(5.3,6.4)$ & $5.6(5.4,6.0)$ & $5.7(5.4,6.2)$ & 0.67 \\
\hline NT-proBNP (mmol/L) & $42(21,67)$ & $64(37,112)$ & $70(39,153)$ & 0.003 \\
\hline
\end{tabular}

Presented as absolute numbers or medians with percentages or quartiles 1 and 3. p-values are for trend. Abbreviations: eGFR ckd-epi-estimated glomerular filtrations rate using the Chronic Kidney Disease Epidemiology Collaboration equation, ACE-i - Angiotensin converting enzyme inhibitor, ARB - Angiotensin-receptor blocker 
3.2 Angiographic coronary artery disease severity and resting concentrations of hs-cTnI and $h s-c \operatorname{Tn} T$

All patients had resting hs-cTnI concentrations above the LoD. Two-hundred-sixty-two (88\%) patients had hs-cTnT concentrations above the LoB (3 ng/L), of which 190 (64\%) had concentrations above the LoD (5 ng/L). When imputing values for hs-cTnT concentrations $<\mathrm{LoB}$, hs-cTnI and hs-cTnT concentrations in the total population as well as in the subset of patients with hs-cTnT concentrations $>$ LoB, correlated significantly $($ Spearman's rho $(r)=$ $0.66, p<0.001$ and $\mathrm{r}=0.60, p<0.001$, respectively). When dichotomizing the $\mathrm{cTn}$ concentrations at the assay-specific $99^{\text {th }}$ percentiles the concordance rate between the two assays was 0.93 (95\% confidence interval [CI] 0.90-0.96), $p<0.001$. Supplemental figure 1 shows a scatterplot visualizing the correlation between concentrations of hs-cTnI and hscTnT.

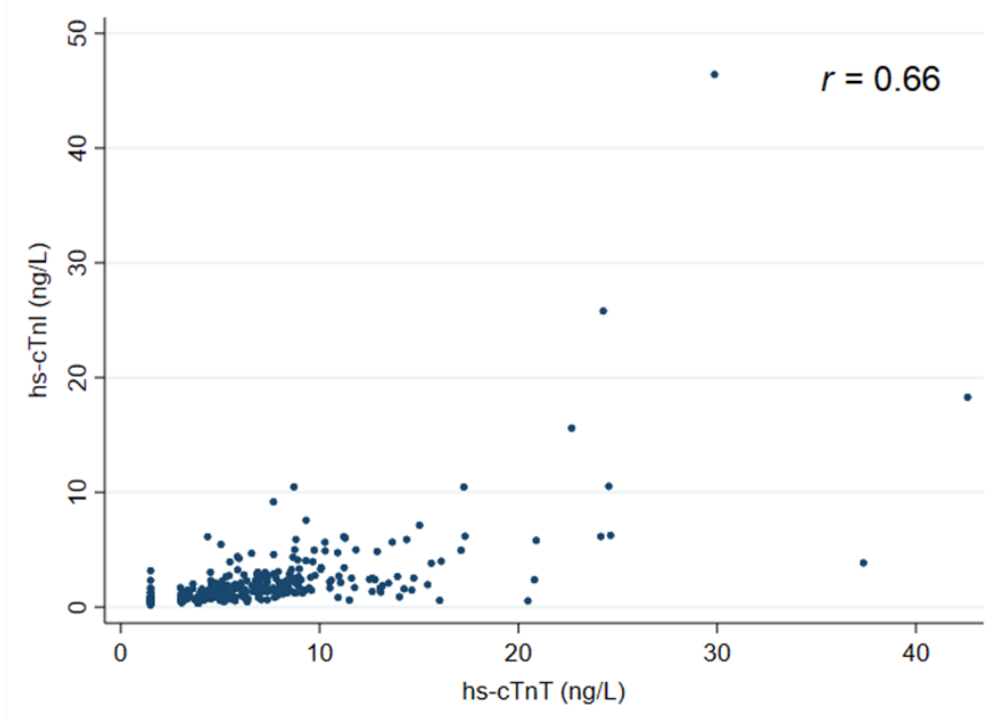

Supplemental figure 1. Scatterplot visualising the correlations between resting cardiac troponin I and T (hs-cTnI; hs-cTnT). Two outliers have been removed in this plot for enhanced readability. 
The median (Q1-3) resting hs-cTnI concentrations in the total population, and in women and men separately, were $1.5(0.9-2.4) \mathrm{ng} / \mathrm{L}, 1.2(0.7-1.7) \mathrm{ng} / \mathrm{L}$ and $1.8(1.0-2.9) \mathrm{ng} / \mathrm{L}$, respectively. In adjusted multivariable linear regression models, older age and male sex were associated with greater concentrations of hs-cTnI while current smoking was associated with lower values. These variables accounted for $19 \%$ of the variance in hs-cTnI (adjusted $\left.R^{2}=0.19\right)$. (Table 2).

Table 2. Linear regression analyses of predictors of resting cardiac troponin I and T (hs-cTnI; hs-cTnT).

\begin{tabular}{|c|c|c|c|c|c|c|c|}
\hline & & \multicolumn{3}{|c|}{ Univariable analyses } & \multicolumn{3}{|c|}{ Multivariable analyses } \\
\hline & & B & $\begin{array}{c}\text { 95\% Confidence } \\
\text { interval }\end{array}$ & p-value & B & $\begin{array}{c}\text { 95\% Confidence } \\
\text { interval }\end{array}$ & p-value \\
\hline \multirow{2}{*}{ Age (years) } & $h s-c T n l$ & 0.44 & $0.29-0.59$ & $<0.001$ & 0.48 & $0.33-0.62$ & $<0.001$ \\
\hline & $h s-c T n T$ & 0.46 & $0.35-0.58$ & $<0.001$ & 0.46 & $0.32-0.59$ & $<0.001$ \\
\hline \multirow{2}{*}{ Male sex } & hs-cTnl & 0.57 & $0.29-0.86$ & $<0.001$ & 0.69 & $0.42-0.97$ & $<0.001$ \\
\hline & $h s-c T n T$ & 0.75 & $0.53-0.98$ & $<0.001$ & 0.92 & $0.73-1.12$ & $<0.001$ \\
\hline \multirow{2}{*}{ Current smoker } & hs-cTnl & -0.62 & $-0.98--0.26$ & 0.001 & -0.40 & $-0.73--0.06$ & 0.02 \\
\hline & hs-cTnT & -0.50 & $-0.80--0.21$ & 0.001 & - & - & NS \\
\hline \multirow{2}{*}{$\begin{array}{l}\text { Body Mass Index } \\
\left(\mathrm{kg} / \mathrm{m}^{2}\right)\end{array}$} & hs-cTnl & 0.01 & $-0.02-0.05$ & 0.52 & - & - & - \\
\hline & hs-cTnT & 0.02 & $-0.006-0.05$ & 0.12 & - & - & - \\
\hline \multirow{2}{*}{ History of diabetes } & hs-cThl & -0.19 & $-0.55-0.17$ & 0.3 & - & - & - \\
\hline & hs-cTnT & 0.13 & $-0.17-0.42$ & 0.39 & - & - & - \\
\hline \multirow{2}{*}{$\begin{array}{l}\text { History of coronary } \\
\text { artery disease }\end{array}$} & hs-cTnl & 0.38 & $0.08-0.69$ & 0.01 & - & - & NS \\
\hline & hs-cTnT & 0.42 & $0.17-0.67$ & 0.001 & - & - & NS \\
\hline \multirow{2}{*}{$\begin{array}{l}\text { Systolic blood } \\
\text { pressure }(\mathrm{mmHg})\end{array}$} & hs-cTnl & 0.12 & $0.05-0.19$ & 0.001 & - & - & NS \\
\hline & $h s-c T n T$ & 0.08 & $0.02-0.14$ & 0.006 & - & - & NS \\
\hline \multirow{2}{*}{$\begin{array}{l}\text { eGFR ckd-epi } \\
\left(\mathrm{ml} / \mathrm{min} / 1.73^{2}\right)\end{array}$} & hs-cTnl & -0.02 & $-0.03--0.01$ & $<0.001$ & - & - & NS \\
\hline & hs-cTnT & -0.02 & $-0.03--0.01$ & $<0.001$ & -0.01 & $-0.02--0.001$ & 0.02 \\
\hline
\end{tabular}

Results presented as unadjusted and adjusted coefficients with 95\% confidence intervals and p-values. Concentrations of cardiac troponin I and T and Gensini score are $\log _{2}$-transformed. Age and systolic blood pressure are reported per 10-unit increase. Abbreviations: hs-cTnl high-sensitivity cardiac troponin I, hs-cTnT - high-sensitivity cardiac troponin T, eGFR ckd-epi - estimated glomerular filtrations rate using the Chronic Kidney Disease Epidemiology Collaboration equation, NS - non-significant 
The median (Q1-3) resting hs-cTnT concentrations in the total population, and in women and men separately were 6 (4-9) ng/L, 5 (3-7) ng/L and 7 (5-10) ng/L, respectively. In adjusted multivariable linear regression models, older age, male sex and lower eGFR were associated with greater concentrations of hs-cTnT. These variables accounted for $36 \%$ of the variance of hs-cTnT (adjusted $R^{2}=0.36$ ).

Supplemental table 1 shows Spearman rank coefficients of correlation between hs-cTnT, hscTnI, demographic, clinical and laboratory variables.

Supplemental Table 1. Spearman rank correlation coefficients between cardiac troponin I (hs-cTnI), cardiac troponin T (hs-cTnT), demographic, clinical and laboratory parameters in the total population.

\begin{tabular}{|c|c|c|c|c|}
\hline & hs-cTnl & p-value & hs-cTnT & p-value \\
\hline Age (years) & 0.33 & $<0.001$ & 0.44 & $<0.001$ \\
\hline Sex & 0.27 & $<0.001$ & 0.33 & $<0.001$ \\
\hline BMI $\left(\mathrm{kg} / \mathrm{m}^{2}\right)$ & 0.04 & 0.47 & 0.07 & 0.26 \\
\hline Heart rate & -0.12 & 0.03 & -0.11 & 0.07 \\
\hline Systolic blood pressure (mmHg) & 0.20 & $<0.001$ & 0.18 & $<0.001$ \\
\hline Current smoking & -0.18 & $<0.001$ & -0.18 & $<0.001$ \\
\hline History of coronary artery disease & 0.19 & $<0.001$ & 0.20 & $<0.001$ \\
\hline History of diabetes type I or II & -0.05 & 0.41 & 0.07 & 0.21 \\
\hline Hemoglobin concentration (g/dl) & 0.18 & $<0.001$ & 0.17 & $<0.001$ \\
\hline White blood cell count $\left(\times 10^{9} / \mathrm{L}\right)$ & -0.01 & 0.92 & 0.09 & 0.13 \\
\hline NT-proBNP (mmol/L) & 0.39 & $<0.001$ & 0.35 & $<0.001$ \\
\hline eGFR ckd-epi $\left(\mathrm{ml} / \mathrm{min} / 1.73^{2}\right)$ & -0.32 & $<0.001$ & -0.32 & $<0.001$ \\
\hline Low density lipoprotein cholesterol ( $\mathrm{mmol} / \mathrm{L})$ & -0.06 & 0.28 & -0.11 & 0.07 \\
\hline Gensini score & 0.37 & $<0.001$ & 0.44 & $<0.001$ \\
\hline hs-cTnl (ng/L) & - & - & 0.66 & $<0.001$ \\
\hline hs-cTnT (ng/L) & 0.66 & $<0.001$ & - & - \\
\hline
\end{tabular}

Abbreviations: eGFR ckd-epi - estimated glomerular filtrations rate using the Chronic Kidney Disease Epidemiology Collaboration equation, hs-cTnl - high-sensitivity cardiac troponin l, hscTnT - high-sensitivity cardiac troponin T 
In patients with no $\mathrm{CAD}$, non-severe $\mathrm{CAD}$ and severe $\mathrm{CAD}$ the median $(\mathrm{Q} 1-3)$ concentrations of hs-cTnI were $1.2(0.7-1.5) \mathrm{ng} / \mathrm{L}, 1.3(0.7-2.1) \mathrm{ng} / \mathrm{L}$ and 1.9 (1.4-3.0) ng/L. (Figure 1, panel A) The corresponding hs-cTnT concentrations were 4 (2-5) ng/L, 5 (3-8) ng/L and 8 (5-11) ng/L. (Figure 1, panel B)
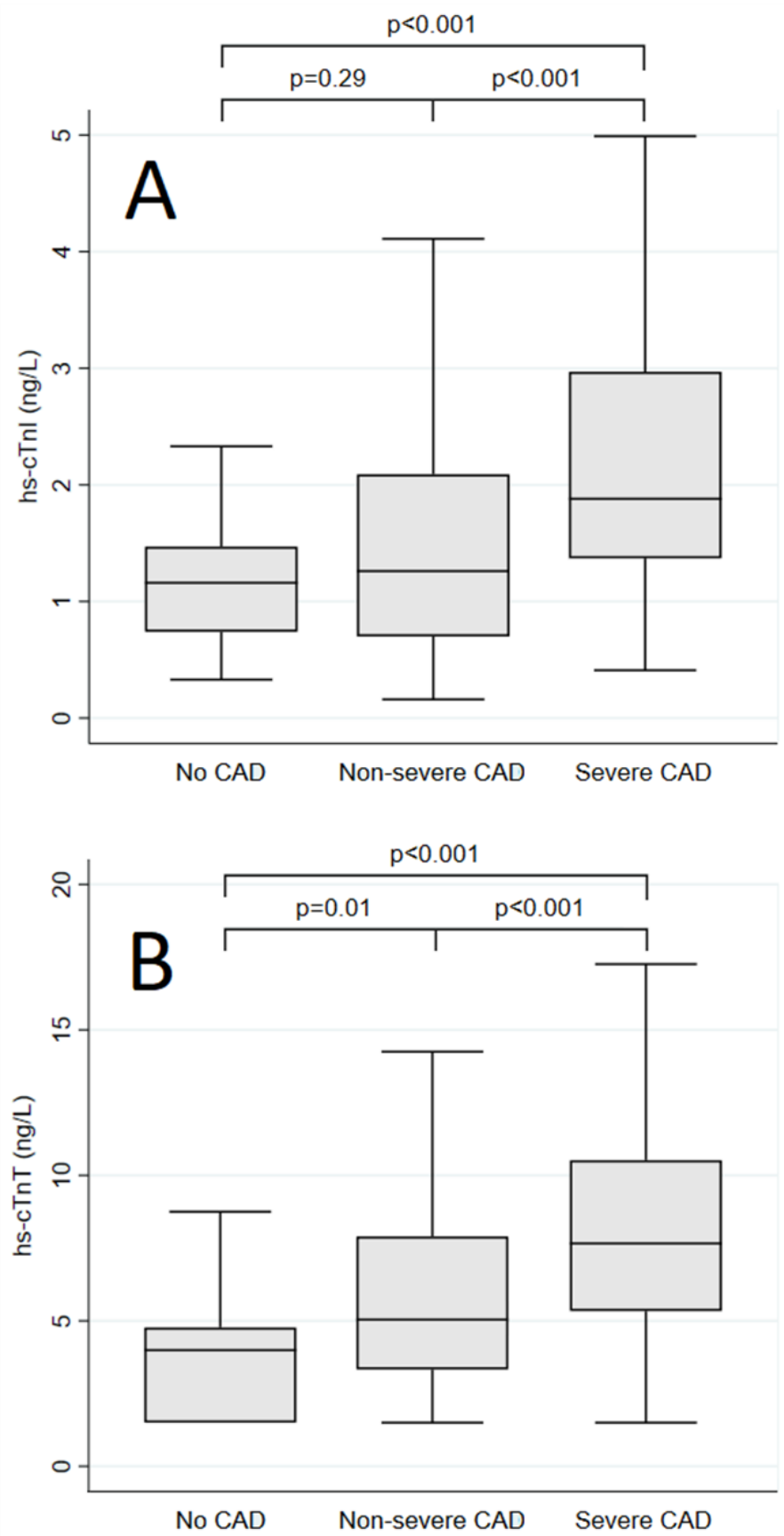

Figure 1. Box plot showing the resting concentrations of hs-cTnI (A) and hs-cTnT $(B)$ in patients stratified by severity of coronary artery disease $(C A D)$. $p$-values are for comparison between two categories. 
Among patients with non-zero Gensini scores ( $\mathrm{n}=272,92 \%)$, both hs-cTnI and hs-cTnT were associated with increasing scores in univariable linear regression analyses (standardized B $0.31,95 \%$ CI [0.20-0.42), $p<0.001]$ and standardized B 0.37, 95\% CI [0.25-0.48), $p<0.001]$, respectively) (For doubling of Gensini score per doubling of hs-cTnI concentrations).

These associations remained significant after adjusting for sex, age, BMI, systolic blood pressure, history of diabetes, current smoking and eGFR. hs-cTnI: Standardized B 0.18, 95\% CI (0.06-0.30), $p=0.002$; hs-cTnT: Standardized B 0.19. 95\% CI (0.09-0.41), $p<0.001$. No significant difference in the strength of the associations was observed ( $p=0.97$ for difference). Both hs-cTnI and hs-cTnT were associated with the presence of severe CAD $(n=137)$ in unadjusted and adjusted logistic regression analyses (hs-cTnI: Unadjusted standardized odds ratio [OR]: 1.88, 95\% CI [1.42-2.47], $p<0.001$, adjusted standardized OR: $1.40,95 \%$ CI (1.05-1.87), $p=0.02$; hs-cTnT: Unadjusted standardized OR: 2.43 [1.80-3.28], $p<0.001$, adjusted standardized OR: 1.96, 95\% CI (1.43-2.70), $p<0.001), p=0.08$ for difference between the adjusted standardized OR.

The ROC-AUC for predicting severe CAD was 0.68 (95\% CI [0.62-0.74]) for resting hs-cTnI and $0.72(0.66-0.78)$ for resting hs-cTnT, $p=0.11$ for difference. When adding resting hs-cTn concentrations to a base risk model for prediction of severe CAD consisting of age, sex, history of CAD, diabetes, hypertension, low-density lipoprotein cholesterol concentrations and current smoking, hs-cTn reclassified patients to a more correct risk stratum (hs-cTnI: cNRI 36\%, 95\% CI [5-65], IDI 1\% [0-8]; hs-cTnT: cNRI 27\% [11-68], IDI 2\% [0-9]). Neither hs-cTnI, nor hs-cTnT significantly increased the c-statistics of the base model $(0.76,95 \% \mathrm{CI}$ [0.70-0.82]). hs-cTnI: 0.76 (0.70-0.82), $p=0.91$; hs-cTnT: 0.77 (0.71-0.83), $p=0.73$. 
The median (Q1-3) absolute change in hs-cTnI concentrations in response to exercise was 0.2 (0.0-0.4) ng/L, corresponding to a relative change of $13(3-27) \%, p<0.001$. Nineteen (7\%) patients had $\geq 2$-fold increase (from median 0.9 (Q1-3 0.6-1.5) ng/L to 2.3 (1.4-5.8) ng/L. (Figure 2).

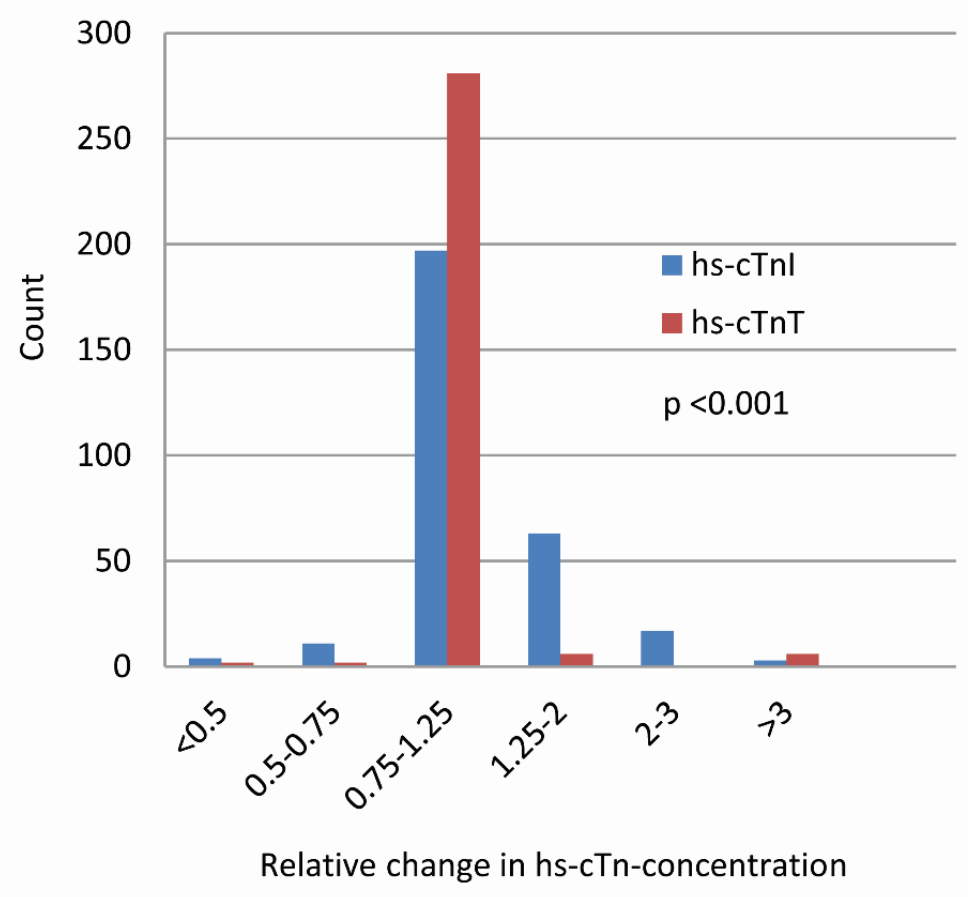

Figure 2. Comparison of the magnitude of relative change in hs-cTnI and hs-cTnT concentrations in response to physical exercise. A value of two corresponds to a doubling relative to resting cTn concentrations.

The median (Q1-3) absolute change in hs-cTnT concentrations was 0 (0-1) ng/L, corresponding to relative change of $5(0-12) \%, p<0.001$. Six $(2 \%)$ patients had $\geq 2$-fold increase (from median 5 (Q1-3 3-8) ng/L to 20 (15-26) ng/L).

There was a significant difference between the exercise-induced relative changes in cTn concentrations between cTnI and cTnT (median 13 (Q1-3 3-27) \% vs. 5 (0-12)\%, 
respectively, $p$ for difference $<0.001)$. In sensitivity analyses only including patients with both pre- and post-test hs-cTnT above the LoB $(\mathrm{n}=260)$ the median $(\mathrm{Q} 1-3)$ cTn relative changes in concentration were 13 (3-26) \% for cTnI and 6 (1-13) \% for cTnT, p for difference $<0.001)$. The correlations between absolute and relative changes in hs-cTnI and hs-cTnT concentrations were $\mathrm{r}=0.13, p=0.03$ and $\mathrm{r}=0.09, p=0.13$, respectively.

We did not identify any clinical predictors, including age, sex and history of CAD, of greater exercise-induced change in either hs-cTnI or hs-cTnT concentrations, with the exception of current smoking, which was associated with a greater relative increase in exercise-induced hscTnI concentrations. (Supplemental Table 2).

Supplemental Table 2. Linear regression analyses of relative increase in cardiac troponin I and T (hs-cTnI; hs-cTnT) in response to exercise.

\begin{tabular}{|c|c|c|c|c|}
\hline & & \multicolumn{3}{|c|}{ Univariable analyses } \\
\hline & & B & $\begin{array}{c}\text { 95\% Confidence } \\
\text { interval }\end{array}$ & p-value \\
\hline \multirow{2}{*}{ Age (years) } & hs-cTnl & -0.06 & $-0.14-0.03$ & 0.20 \\
\hline & hs-cTnT & 0.003 & $-0.04-0.05$ & 0.88 \\
\hline \multirow{2}{*}{ Female sex } & hs-cTnl & -0.13 & $-0.29-0.03$ & 0.12 \\
\hline & hs-cTnT & -0.06 & $-0.14-0.02$ & 0.16 \\
\hline \multirow{2}{*}{ Current smoker } & hs-cTnl & 0.28 & $0.08-0.48$ & 0.005 \\
\hline & hs-cTnT & 0.009 & $-0.09-0.11$ & 0.86 \\
\hline \multirow{2}{*}{ Body Mass Index (kg/m²) } & hs-cTnl & -0.01 & $-0.03-0.005$ & 0.15 \\
\hline & hs-cTnT & 0.007 & $-0.003-0.07$ & 0.16 \\
\hline \multirow{2}{*}{ History of diabetes } & hs-cTnl & -0.08 & $-0.27-0.12$ & 0.45 \\
\hline & hs-cTnT & 0.06 & $-0.04-0.16$ & 0.25 \\
\hline \multirow{2}{*}{ History of coronary artery disease } & hs-cTnl & -0.03 & $-0.20-0.13$ & 0.70 \\
\hline & hs-cTnT & 0.04 & $-0.04-0.13$ & 0.32 \\
\hline \multirow{2}{*}{ Systolic blood pressure (mmHg) } & $h s-c T n l$ & 0.02 & $-0.02-0.05$ & 0.42 \\
\hline & hs-cTnT & -0.003 & $-0.02-0.02$ & 0.73 \\
\hline \multirow{2}{*}{ eGFR ckd-epi (ml/min/1.73²) } & hs-cTnl & 0.003 & $-0.002-0.01$ & 0.23 \\
\hline & hs-cTnT & 0.0006 & $-0.002-0.003$ & 0.93 \\
\hline \multirow{2}{*}{ Gensini score } & $h s-c T n l$ & 0.02 & $-0.03-0.07$ & 0.37 \\
\hline & hs-cTnT & 0.001 & $-0.02-0.03$ & 0.93 \\
\hline \multirow{2}{*}{ METs } & hs-cTnl & 0.01 & $-0.03-0.06$ & 0.52 \\
\hline & $h s-c T n T$ & 0.004 & $-0.02-0.03$ & 0.72 \\
\hline \multirow{2}{*}{ Exercise duration (minutes) } & $h s-c T n l$ & -0.008 & $-0.03-0.01$ & 0.46 \\
\hline & hs-cTnT & 0.007 & $-0.004-0.02$ & 0.21 \\
\hline
\end{tabular}


Results presented as unadjusted coefficients with 95\% confidence intervals and p-values. Concentrations of cardiac troponin I and T, Gensini score and the relative cTn increase are $\log _{2}$-transformed. Age and systolic blood pressure are reported per 10-unit increase. Abbreviations: hs-cTnl - high-sensitivity cardiac troponin I, hs-cTnT - high-sensitivity cardiac troponin T, eGFR ckd-epi-estimated glomerular filtrations rate using the Chronic Kidney Disease Epidemiology Collaboration equation

There was no significant association between relative changes in hs-cTnI and hs-cTnT concentrations and Gensini score ( $p=0.37$ and $p=0.93$, respectively). Moreover, relative changes in hs-cTnI and hs-cTnT during exercise did not differ between participants with no CAD, non-severe CAD and severe CAD (Supplemental Table 3). The ROC-AUC for relative changes in hs-cTnI and hs-cTnT concentrations in association with severe CAD was $0.50(0.43-0.57)$ and $0.53(0.46-0.60)$, respectively.

Supplemental Table 3. Exercise characteristics, resting cardiac troponin values and relative increase in response to exercise stratified by categories of Gensini score.

\begin{tabular}{|l|c|c|c|c|}
\hline & No CAD (Gensini = 0) & $\begin{array}{c}\text { Minimal CAD } \\
\text { (Gensini = 1-19) } \\
\mathbf{n = 1 3 5}\end{array}$ & $\begin{array}{c}\text { Severe CAD (Gensini } \\
\mathbf{2 2 0}) \\
\mathbf{n = 1 3 7}\end{array}$ & $\begin{array}{c}\mathbf{p} \text {-value } \\
\text { for trend }\end{array}$ \\
\hline hs-cTnl at rest (ng/L) & $1.16(0.74,1.47)$ & $1.26(0.7,2.09)$ & $1.88(1.37,2.97)$ & $<0.001$ \\
\hline hs-cTnl delta ratio & $1.18(1.09,1.24)$ & $1.11(1.03,1.28)$ & $1.13(1.03,1.27)$ & 0.63 \\
\hline hs-cTnT at rest (ng/L) & $4(4,5)$ & $5(3,8)$ & $8(5,11)$ & $<0.001$ \\
\hline hs-cTnT delta ratio & $1.03(1.00,1.11)$ & $1.05(1.00,1.13)$ & $1.06(1.01,1.12)$ & 0.31 \\
\hline Exercise duration (min) & $10.3(8.7,12.7)$ & $9.7(7.8,12.2)$ & $9.2(6.6,11.9)$ & 0.02 \\
\hline METs & $7.0(6.3,7.8)$ & $6.9(5.8,8.0)$ & $6.3(5.5,8.0)$ & 0.04 \\
\hline
\end{tabular}

Presented as medians with quartiles 1 and 3. p-values are for trend. Abbreviations: hs-cTnl high-sensitivity cardiac troponin I, hs-cTnT - high-sensitivity cardiac troponin $T$ 


\section{DISCUSSION}

\subsection{Main findings}

There are three main findings in our study investigating the relationship between resting and exercise-induced cTnI and cTnT concentrations and coronary artery disease in patients with suspected CCS.

1) There was a graded relationship between concentrations of both for $\mathrm{cTnI}$ and cTnT at rest and the severity of CAD with no significant differences between the two isotypes.

2) The addition of resting cTn concentrations to a diagnostic model of known predictors of CAD did not significantly enhance the model's diagnostic power, although resting cTn concentrations might provide some reclassification ability.

3) Exercise-induced changes in cTn concentrations were not associated with the presence or severity of CAD, even with the enhanced analytical sensitivity of the cTnI assay. Further, we did not identify any relevant clinical predictors of greater exercise-induced changes in cTn concentrations. Although of no diagnostic significance, the increase in cTn concentrations was greater for $\mathrm{cTnI}$ than for $\mathrm{cTnT}$

\section{2 cTn and myocardial damage}

With the increasing utilization of high-sensitivity cTn assays in the last decade, cTn as a biomarker is now understood to not only reflect the presence of ischemia driven acute myocardial necrosis, but also chronic and subclinical disease processes. In support of this, the fourth universal definition of myocardial infarction defines elevated concentrations of cTn in the non-ischemic setting as either chronic or acute myocardial damage. [1]

To what extent the elevated cTn concentrations observed in patients with chronic myocardial damage represent underlying coronary artery pathology or other pathological processes such 
as left ventricular (LV) remodelling or chronic kidney disease, is not thoroughly understood. Indeed, LV mass is perhaps the most important determinant of cTn concentrations [21], but greater cTnT concentrations also appears to be independently associated with reversible ischemia in patients with CCS. [22] Furthermore, cTnI is associated with the presence of obstructive CAD and coronary artery calcium score in patients with suspected CCS. [2] Our findings extend these observations to a higher risk population (Morise score $>9$ ) and also demonstrate the performance equivalency of cTnI and cTnT, measured with the highest sensitivity assays available. Thus, despite mounting evidence suggesting idiosyncratic biological properties of the cTnI and cTnT isotypes, the association with chronic CAD seems to be comparable.

\subsection{Exercise intensity and temporal release kinetics}

Recent studies have demonstrated that exercised-induced increases in cTn concentrations are associated with increased cardiovascular risk. [9] In an observational study of 725 older individuals participating in a long-distance long-duration march, exercise-induced increases in cTnI concentration above the $99^{\text {th }}$ percentile URL independently predicted higher all-cause mortality and incidence of cardiovascular events. In a heterogenous and comorbid population, these findings challenges the paradigm of the benign nature of post exercise cTn elevations. In our study, cTn concentrations after a traditional high-intensity short-duration bicycle cardiac stress test with immediate post-test sampling of cTn did not associate with coronary pathology. However, many patients had increases in cTn concentrations in response to exercise (a doubling of cTnI and cTnT concentrations were observed in $7 \%$ and $2 \%$ of patients, respectively), but this was not more prevalent in patients with obstructive CAD. This lack of association may represent a benign response to increased contractility and heart rate. However, the limited duration of exercise and short time to sampling may have influenced the results, as both exercise intensity [23] and temporal aspects [24] of cTn kinetics have 
diagnostic and prognostic importance. In a study of 120 middle aged participants in a highintensity mountain bicycle race, post-exercise cTn elevations were significantly associated with the presence of Coronary Computed Tomography Angiography (CCTA) verified CAD. [25] Notably, while both cTnI and cTnT concentrations peaked around 3 hours after exercise, any significant diagnostic utility was only found in samples obtained $24 \mathrm{~h}$ post-exercise. Considering this, the lack of a significant diagnostic signal in our study is not surprising, as our post-exercise samples were obtained immediately after terminations of exercise. While patients in our study performed at a high peak level of intensity, the duration of exercise was limited, resulting in an overall lower burden of physiological stress. Although it is apparent that both intensity and duration of exercise are determinants of inducible cTn concentrations, shorter, high-intensity performance might mimic the physiological stress seen in clinical scenarios such as self-terminating cardiac arrhythmias or episodes of convulsion. As such, our study indicates the low clinical feasibility of repurposing traditional cardiac stress tests for a biomarker driven diagnostic approach, as well as the difficulties associated with interpreting cTn in the setting of acute myocardial damage.

\subsection{Isotype-dependent cTn kinetics}

Although of uncertain clinical significance, emerging evidence indicate that cTnI and cTnT are differentially associated with both physiological processes and clinical outcomes. With cardiomyocyte damage, cTnI seems to be released earlier and in greater magnitude than cTnT [12] and is also less affected by impaired renal function. [15] Concentrations of cTnT appear to have a diurnal variation that is not apparent for $\mathrm{cTnI}[14]$ and also appear more prone to interference by neuromuscular disorders. [26] Prognostically, cTnT appears more strongly associated with all-cause mortality in the general population [10] and after MI [12], while cTnI seems to be more strongly associated with incident coronary heart disease. [27, 28] Furthermore, $\mathrm{cTnI}$ and cTnT appear to provide complementary prognostic information in the 
general population and in patients with CCS. [5, 27] In a situation of MI, cTnI and cTnT concentrations are strongly correlated and as such does not seem to provide substantial complementary diagnostic information as to rule-in MI. However, differences in isotype specific release kinetics could translate into differences in earlier rule-out of MI, as cTnI appears to be a more sensitive marker of myocardial damage at an earlier time point than cTnT. $[12,29]$ In our study, the magnitude of cTnI release in response to physiological stress was indeed significantly greater than that of cTnT, although this did not yield any additional diagnostic information. Whether this reflects true biological differences between the isotypes or if it is related to biochemical properties of the assays utilized in the study is unknown, but knowledge of the phenomenon could be useful for clinicians interpreting cTn concentrations in patients admitted with chest pain after physical activity.

\subsection{Limitations}

The diagnostic precision of a biomarker is dependent on the accuracy of the gold standard definition for the disease being investigated. In our study, all patients underwent invasive coronary angiography and the extent and severity of CAD was estimated using the Gensini score. However, it is uncertain to what extent a pure anatomical estimate of CAD burden is physiologically significant. As such, the lack of measures such as fractional flow reserve (FFR) may attenuate the diagnostic efficacy of cTn and is a major limitation to the study. Furthermore, as cTn is associated with myocardial remodelling, the lack of echocardiographic data limits our ability to control for any effects of subclinical heart failure. However, the low NT-proBNP concentrations (median 64 [Q1-3 37-123] ng/L) suggests that subclinical HF was rare. Further, our study did not include serial sampling of cTn concentrations, and as such does not contain the information needed to opine on the temporal release patterns observed in other studies. [23, 25] Obtaining a single post-exercise sample is a clinically desirable practice, as prompt sampling eliminates the need for prolonged observation. However, it is 
evident that the temporal aspect of cTn release is important and the lack of such data is a limitation to our study. 


\section{CONCLUSION}

There was a graded relationship between resting concentrations of cTn measured with highsensitivity assays and the extent and severity of CAD in patients referred for evaluation of suspected CCS. The clinical and demographic determinants of this relationship were similar for $\mathrm{cTnI}$ and $\mathrm{cTnT}$, despite the superior analytical sensitivity of the cTnI assay. cTnI concentrations increased by a larger magnitude than cTnT concentrations in response to shortduration high-intensity exercise. However, elevation in neither isotype was associated with the presence or severity of CAD. Thus, there appears to be a threshold with regards to exercise intensity and timing of cTn sampling that needs to be exceeded for cTn dynamics to reflect the presence of CAD. 


\section{GLOSSARY}

ACE-i - Angiotensin converting enzyme inhibitor

ACS - Acute Coronary Syndrome

AMI - Acute myocardial infarction

ARB - Angiotensin-receptor blocker

BMI - Body Mass Index

CABG - Coronary artery bypass graft

CAD - Coronary artery disease

CADENCE - Markers of Coronary Artery Disease During Exercise Testing

CCTA - Coronary Computed Tomography Angiography

CI - Confidence Interval

CKD-EPI - Chronic Kidney Disease Epidemiology Collaboration equation

cNRI - Continuous Net Reclassification Improvement

CSS - Chronic Coronary Syndrome

cTnI - Cardiac troponin, isotype I

cTnT - Cardiac troponin, isotype T

CV - Coefficient of variation

eGFR - Estimated glomerular filtration rate

FFR - Fractional Flow Reserve

HF - Heart failure

hs-cTn - High-sensitivity cardiac troponin

IDI - Integrated Discrimination Improvement

LDL cholesterol - Low density lipoprotein cholesterol

LoB - Limit of Blank

LoD - Limit of Detection 
LV - Left ventricle

MET - Metabolic Equivalent of Task

OR - Odds ratio

OUH - Oslo University Hospital

ROC-AUC- Receiver Operating Characteristics - Area Under the Curve

URL - Upper Reference Limit 


\section{REFERENCES}

[1] K. Thygesen, J.S. Alpert, A.S. Jaffe, B.R. Chaitman, J.J. Bax, D.A. Morrow, H.D. White, I. Executive Group on behalf of the Joint European Society of Cardiology /American College of Cardiology /American Heart Association /World Heart Federation Task Force for the Universal Definition of Myocardial, Fourth Universal Definition of Myocardial Infarction (2018), J Am Coll Cardiol 72(18) (2018) 2231-2264.

[2] J.L. Januzzi, Jr., S. Suchindran, A. Coles, M. Ferencik, M.R. Patel, U. Hoffmann, G.S. Ginsburg, P.S. Douglas, P. Investigators, High-Sensitivity Troponin I and Coronary

Computed Tomography in Symptomatic Outpatients With Suspected CAD: Insights From the PROMISE Trial, JACC Cardiovasc Imaging 12(6) (2019) 1047-1055.

[3] N. Reckord, M. Behnes, M. Natale, A. Mukherji, J. Rusnak, U. Ansari, S. Lang, U. Hoffmann, M. Borggrefe, T. Henzler, M. Neumaier, M.T. Kittel, K. Mashayekhi, T. Bertsch, I. Akin, High Sensitivity Troponin I and T Reflect the Presence of Obstructive and MultiVessel Coronary Artery Disease Being Assessed by Coronary Computed Tomography Angiography, Curr Pharm Biotechnol 18(6) (2017) 508-515.

[4] J.L. Januzzi, Jr., S. Suchindran, U. Hoffmann, M.R. Patel, M. Ferencik, A. Coles, J.C. Tardif, G.S. Ginsburg, P.S. Douglas, P. Investigators, Single-Molecule hsTnI and Short-Term Risk in Stable Patients With Chest Pain, J Am Coll Cardiol 73(3) (2019) 251-260.

[5] T. Omland, M.A. Pfeffer, S.D. Solomon, J.A. de Lemos, H. Rosjo, J. Saltyte Benth, A. Maggioni, M.J. Domanski, J.L. Rouleau, M.S. Sabatine, E. Braunwald, P. Investigators, Prognostic value of cardiac troponin I measured with a highly sensitive assay in patients with stable coronary artery disease, J Am Coll Cardiol 61(12) (2013) 1240-9.

[6] F.S. Apple, Y. Sandoval, A.S. Jaffe, J. Ordonez-Llanos, I.T.F.o.C.A.o.C. Bio-Markers, Cardiac Troponin Assays: Guide to Understanding Analytical Characteristics and Their Impact on Clinical Care, Clin Chem 63(1) (2017) 73-81.

[7] P. Baker, T. Leckie, D. Harrington, A. Richardson, Exercise-induced cardiac troponin elevation: An update on the evidence, mechanism and implications, Int J Cardiol Heart Vasc 22 (2019) 181-186.

[8] O. Skadberg, O. Kleiven, M. Bjorkavoll-Bergseth, T. Melberg, R. Bergseth, J. Selvag, B. Auestad, O.J. Greve, K. Dickstein, T. Aarsland, S. Orn, Highly increased Troponin I levels following high-intensity endurance cycling may detect subclinical coronary artery disease in presumably healthy leisure sport cyclists: The North Sea Race Endurance Exercise Study (NEEDED) 2013, Eur J Prev Cardiol 24(8) (2017) 885-894.

[9] V.L. Aengevaeren, M.T.E. Hopman, P.D. Thompson, E.A. Bakker, K.P. George, D.H.J. Thijssen, T.M.H. Eijsvogels, Exercise-Induced Cardiac Troponin I Increase and Incident Mortality and Cardiovascular Events, Circulation 140(10) (2019) 804-814.

[10] P. Welsh, D. Preiss, C. Hayward, A.S.V. Shah, D. McAllister, A. Briggs, C. Boachie, A. McConnachie, S. Padmanabhan, C. Welsh, M. Woodward, A. Campbell, D. Porteous, N.L. Mills, N. Sattar, Cardiac Troponin T and Troponin I in the General Population, Circulation 139(24) (2019) 2754-2764.

[11] P. Welsh, D. Preiss, A.S.V. Shah, D. McAllister, A. Briggs, C. Boachie, A.

McConnachie, C. Hayward, S. Padmanabhan, C. Welsh, M. Woodward, A. Campbell, D. Porteous, N.L. Mills, N. Sattar, Comparison between High-Sensitivity Cardiac Troponin T and Cardiac Troponin I in a Large General Population Cohort, Clin Chem 64(11) (2018) 1607-1616.

[12] M. Rubini Gimenez, R. Twerenbold, T. Reichlin, K. Wildi, P. Haaf, M. Schaefer, C. Zellweger, B. Moehring, F. Stallone, S.M. Sou, M. Mueller, K. Denhaerynck, T. Mosimann, M. Reiter, B. Meller, M. Freese, C. Stelzig, I. Klimmeck, J. Voegele, B. Hartmann, K. 
Rentsch, S. Osswald, C. Mueller, Direct comparison of high-sensitivity-cardiac troponin I vs. T for the early diagnosis of acute myocardial infarction, Eur Heart J 35(34) (2014) 2303-11. [13] S.H. Tveit, P.L. Myhre, N.J.S. Hoff, T.M. Le, I. Seljeflot, R. Roysland, A.D. Hoiseth, H. Rosjo, T. Omland, Superiority of high sensitivity cardiac troponin T vs. I for long-term prognostic value in patients with chest pain; data from the Akershus cardiac Examination (ACE) 3 study, Clin Biochem 78 (2020) 10-17.

[14] L.J. Klinkenberg, K. Wildi, N. van der Linden, I.W. Kouw, M. Niens, R. Twerenbold, M. Rubini Gimenez, C. Puelacher, J. Daniel Neuhaus, P. Hillinger, T. Nestelberger, J. Boeddinghaus, K. Grimm, Z. Sabti, J.A. Bons, J.D. van Suijlen, F.E. Tan, J. Ten Kate, O. Bekers, L.J. van Loon, M.P. van Dieijen-Visser, C. Mueller, S.J. Meex, Diurnal Rhythm of Cardiac Troponin: Consequences for the Diagnosis of Acute Myocardial Infarction, Clin Chem 62(12) (2016) 1602-1611.

[15] F. Artunc, C. Mueller, T. Breidthardt, R. Twerenbold, A. Peter, C. Thamer, P. Weyrich, H.U. Haering, B. Friedrich, Sensitive troponins--which suits better for hemodialysis patients? Associated factors and prediction of mortality, PLoS One 7(10) (2012) e47610.

[16] J. Cwikiel, I. Seljeflot, E. Berge, H. Arnesen, K. Wachtell, H. Ulsaker, A. Flaa, Procoagulant activity during exercise testing in patients with coronary artery disease, Thromb $\mathrm{J}$ 15 (2017) 3.

[17] A.P. Morise, W.J. Haddad, D. Beckner, Development and validation of a clinical score to estimate the probability of coronary artery disease in men and women presenting with suspected coronary disease, Am J Med 102(4) (1997) 350-6.

[18] G.A. Borg, Psychophysical bases of perceived exertion, Med Sci Sports Exerc 14(5) (1982) 377-81.

[19] W.G. Austen, J.E. Edwards, R.L. Frye, G.G. Gensini, V.L. Gott, L.S. Griffith, D.C. McGoon, M.L. Murphy, B.B. Roe, A reporting system on patients evaluated for coronary artery disease. Report of the Ad Hoc Committee for Grading of Coronary Artery Disease, Council on Cardiovascular Surgery, American Heart Association, Circulation 51(4 Suppl) (1975) 5-40.

[20] G.G. Gensini, A more meaningful scoring system for determining the severity of coronary heart disease, Am J Cardiol 51(3) (1983) 606.

[21] J.A. de Lemos, M.H. Drazner, T. Omland, C.R. Ayers, A. Khera, A. Rohatgi, I. Hashim, J.D. Berry, S.R. Das, D.A. Morrow, D.K. McGuire, Association of troponin T detected with a highly sensitive assay and cardiac structure and mortality risk in the general population, JAMA 304(22) (2010) 2503-12.

[22] P.L. Myhre, T. Omland, S.I. Sarvari, H. Ukkonen, F. Rademakers, J.E. Engvall, T.A. Hagve, E. Nagel, R. Sicari, J.L. Zamorano, M. Monaghan, J. D'Hooge, T. Edvardsen, H. Rosjo, D.-C.S. Group, Cardiac Troponin T Concentrations, Reversible Myocardial Ischemia, and Indices of Left Ventricular Remodeling in Patients with Suspected Stable Angina Pectoris: a DOPPLER-CIP Substudy, Clin Chem 64(9) (2018) 1370-1379.

[23] G.M. Stewart, A. Yamada, L.J. Haseler, J.J. Kavanagh, J. Chan, G. Koerbin, C. Wood, S. Sabapathy, Influence of exercise intensity and duration on functional and biochemical perturbations in the human heart, J Physiol 594(11) (2016) 3031-44.

[24] A.T. Turer, T.A. Addo, J.L. Martin, M.S. Sabatine, G.D. Lewis, R.E. Gerszten, E.C. Keeley, J.E. Cigarroa, R.A. Lange, L.D. Hillis, J.A. de Lemos, Myocardial ischemia induced by rapid atrial pacing causes troponin $T$ release detectable by a highly sensitive assay: insights from a coronary sinus sampling study, J Am Coll Cardiol 57(24) (2011) 2398-405.

[25] O. Kleiven, T. Omland, O. Skadberg, T.H. Melberg, M.F. Bjorkavoll-Bergseth, B. Auestad, R. Bergseth, O.J. Greve, K.M. Aakre, S. Orn, Occult obstructive coronary artery disease is associated with prolonged cardiac troponin elevation following strenuous exercise, Eur J Prev Cardiol 27(11) (2020) 1212-1221. 
[26] D. Rittoo, A. Jones, B. Lecky, D. Neithercut, Elevation of cardiac troponin T, but not cardiac troponin I, in patients with neuromuscular diseases: implications for the diagnosis of myocardial infarction, J Am Coll Cardiol 63(22) (2014) 2411-20.

[27] X. Jia, W. Sun, R.C. Hoogeveen, V. Nambi, K. Matsushita, A.R. Folsom, G. Heiss, D.J. Couper, S.D. Solomon, E. Boerwinkle, A. Shah, E. Selvin, J.A. de Lemos, C.M. Ballantyne, High-Sensitivity Troponin I and Incident Coronary Events, Stroke, Heart Failure Hospitalization, and Mortality in the ARIC Study, Circulation 139(23) (2019) 2642-2653. [28] R.M. Oemrawsingh, K.M. Akkerhuis, M. de Mulder, V.A. Umans, B. Kietselaer, C. Schotborgh, E. Ronner, T. Lenderink, A. Liem, D. Haitsma, P. van der Harst, F.W. Asselbergs, A. Maas, A.J. Oude Ophuis, B. Ilmer, R. Dijkgraaf, R.J. de Winter, S.H. Kie The, A.J. Wardeh, W. Hermans, E. Cramer, R.H. van Schaik, I.E. Hoefer, P.A. Doevendans, M.L. Simoons, E. Boersma, B.I. Investigators, High-Frequency Biomarker Measurements of Troponin, NT-proBNP, and C-Reactive Protein for Prediction of New Coronary Events After Acute Coronary Syndrome, Circulation 139(1) (2019) 134-136.

[29] N. van der Linden, K. Wildi, R. Twerenbold, J.W. Pickering, M. Than, L. Cullen, J. Greenslade, W. Parsonage, T. Nestelberger, J. Boeddinghaus, P. Badertscher, M. Rubini Gimenez, L.J.J. Klinkenberg, O. Bekers, A. Schoni, D.I. Keller, Z. Sabti, C. Puelacher, J. Cupa, L. Schumacher, N. Kozhuharov, K. Grimm, S. Shrestha, D. Flores, M. Freese, C. Stelzig, I. Strebel, O. Miro, K. Rentsch, B. Morawiec, D. Kawecki, W. Kloos, J. Lohrmann, A.M. Richards, R. Troughton, C. Pemberton, S. Osswald, M.P. van Dieijen-Visser, A.M. Mingels, T. Reichlin, S.J.R. Meex, C. Mueller, Combining High-Sensitivity Cardiac Troponin I and Cardiac Troponin $\mathrm{T}$ in the Early Diagnosis of Acute Myocardial Infarction, Circulation 138(10) (2018) 989-999. 